\title{
Signaled two-way avoidance learning using electrical stimulation of the inferior colliculus as negative reinforcement: effects of visual and auditory cues as warning stimuli
}
A.C. Troncoso',
G. Cirilo-Júnior ${ }^{1}$,
G. Sandner ${ }^{2}$ and M.L. Brandão ${ }^{1}$

\author{
${ }^{1}$ Laboratório de Psicobiologia, Departamento de Psicologia, Faculdade de Filosofia, \\ Ciências e Letras de Ribeirão Preto, Universidade de São Paulo, Ribeirão Preto, SP, \\ Brasil \\ 'Laboratoire de Psychopathologie et Psychopharmacologie de la Cognition, U405 \\ INSERM, Strasbourg, France
}

\section{Correspondence \\ M.L. Brandão \\ Laboratório de Psicobiologia \\ Departamento de Psicologia \\ Faculdade de Filosofia, Ciências \\ e Letras de Ribeirão Preto, USP \\ Av. Bandeirantes, 3900 \\ 14049-901 Ribeirão Preto, SP \\ Brasil}

Research supported by FAPESP (No. 94/5933-2). A.C. Troncoso was the recipient of a FAPESP scholarship and G. Cirilo was the recipient of a scientific initiation scholarship from CNPq. This work is part of a

Brazil-France exchange program (CAPES/COFECUB No. 164/94).

Received February 24, 1997 Accepted December 17, 1997

\section{Abstract}

The inferior colliculus is a primary relay for the processing of auditory information in the brainstem. The inferior colliculus is also part of the so-called brain aversion system as animals learn to switch off the electrical stimulation of this structure. The purpose of the present study was to determine whether associative learning occurs between aversion induced by electrical stimulation of the inferior colliculus and visual and auditory warning stimuli. Rats implanted with electrodes into the central nucleus of the inferior colliculus were placed inside an open-field and thresholds for the escape response to electrical stimulation of the inferior colliculus were determined. The rats were then placed inside a shuttle-box and submitted to a two-way avoidance paradigm. Electrical stimulation of the inferior colliculus at the escape threshold $(98.12 \pm 6.15$ (A, peak-to-peak) was used as negative reinforcement and light or tone as the warning stimulus. Each session consisted of 50 trials and was divided into two segments of 25 trials in order to determine the learning rate of the animals during the sessions. The rats learned to avoid the inferior colliculus stimulation when light was used as the warning stimulus $(13.25 \pm 0.60 \mathrm{~s}$ and 8.63 $\pm 0.93 \mathrm{~s}$ for latencies and $12.5 \pm 2.04$ and $19.62 \pm 1.65$ for frequencies in the first and second halves of the sessions, respectively, $\mathrm{P}<0.01$ in both cases). No significant changes in latencies (14.75 \pm 1.63 and $12.75 \pm 1.44 \mathrm{~s})$ or frequencies of responses $(8.75 \pm 1.20$ and $11.25 \pm$ 1.13) were seen when tone was used as the warning stimulus $(\mathrm{P}>0.05$ in both cases). Taken together, the present results suggest that rats learn to avoid the inferior colliculus stimulation when light is used as the warning stimulus. However, this learning process does not occur when the neutral stimulus used is an acoustic one. Electrical stimulation of the inferior colliculus may disturb the signal transmission of the stimulus to be conditioned from the inferior colliculus to higher brain structures such as amygdala.
Key words

- Inferior colliculus

- Active avoidance

- Selective attention

...................... 


\section{Introduction}

In addition to being an important relay station for auditory information in the brainstem, the inferior colliculus also plays an important role in the mediation of defensive behaviors together with the amygdala, medial hypothalamus, dorsal periaqueductal gray matter and deep layers of the superior colliculus $(1,2)$. Evidence for the involvement of the inferior colliculus in the elaboration of aversive states has been provided by both immunohistochemical and behavioral data (3-5). C-fos immunoreactivity studies have shown that this structure is also labeled along with the amygdala, hypothalamus and dorsal periaqueductal gray matter following either electrical or chemical stimulation of the latter structure or exposure of the animals to aversive environmental stimulation (3). Based on behavioral data, it has been shown that electrical or chemical stimulation of the inferior colliculus produces flight behavior and other fear-like responses (4-6). Furthermore, microinjection of bicuculline, a GABA-A antagonist, into the inferior colliculus produces a behavioral activation, together with autonomic responses, similar to the defense reaction induced by electrical or chemical stimulation of other sites in the brain aversion system (7). It has been suggested that a pathway through the inferior colliculus to the medial geniculate body is involved in tone conditioning and that the emotional processing depends upon afferent information from this thalamic region to the amygdala $(8,9)$.

Recent evidence obtained in our laboratory has demonstrated that pairing visual stimulation and aversive electrical stimulation of the inferior colliculus (10) produces associative learning. Thus, the inferior colliculus appears to be involved in linking external stimuli and aversiveness at the brainstem level. Since the inferior colliculus is primarily involved in the processing of auditory information, the present study addressed the question of whether this property holds true regardless of the type of sensory stimulation (auditory or visual) used as the neutral stimulus. This approach may be important for the understanding of the processing of sensory information of different modalities and for the assessment of basic mechanisms of selective attention (11). To examine this issue, we used a two-way active avoidance procedure in which light or tone was used as a signal warning about the onset of electrical stimulation of the inferior colliculus. Thus, electrical stimulation of the inferior colliculus was the unconditioned stimulus paired with the houselight or a tone as the warning stimulus.

\section{Material and Methods}

\section{Animals}

Twenty-four male Wistar rats weighing 250-300 $\mathrm{g}$ were housed in individual Plexiglas-walled cages under a 12:12 h dark/ light cycle (lights on at 6:00 a.m.) at $23 \pm$ $1^{\circ} \mathrm{C}$, with free access to food and water throughout the experiment.

\section{Surgery}

The animals were anesthetized with sodium pentobarbital (45 mg/kg, ip) and fixed in a stereotaxic frame (David Kopf, Tujunga, CA). A bipolar stainless steel electrode (Plastic Products MS 303/2, Roanoke, VA) was stereotaxically implanted into the midbrain, aimed at the inferior colliculus. The electrode was made of stainless steel wire, 160 $\mu \mathrm{m}$ in diameter, insulated except at the crosssection of the tip. The upper incisor bar was set at $3.3 \mathrm{~mm}$ below the interaural line, so that the skull was horizontal between bregma and lambda. The electrode was introduced vertically using the following coordinates with the lambda serving as the reference for each plane: antero-posterior, $-1.2 \mathrm{~mm}$; mediolateral, $1.5 \mathrm{~mm}$, and dorsoventral, 4.5 
$\mathrm{mm}$ (12). The electrode was fixed to the skull by means of acrylic resin and three stainless steel screws. The electrode wire was connected to an amphenol socket at the end of a flexible electrical cable and used for brain stimulation.

\section{Apparatus and procedure}

One week after surgery, the rats were placed in an open-field, which was a circular enclosure $60 \mathrm{~cm}$ in diameter and $50 \mathrm{~cm}$ high and allowed to habituate to the enclosure for 15 min. The brain was stimulated electrically with a sine wave stimulator (13). The stimulation current was monitored by measuring the voltage drop across a $1 \mathrm{~K}$ resistor with an oscilloscope (Labo, São Paulo, Brazil). Brain stimulation with alternating current $(\mathrm{AC} ; 60 \mathrm{~Hz})$ for $15 \mathrm{~s}$ was presented at 1 min intervals with the current intensity increasing by steps of $5 \mu \mathrm{A}$ (peak-to-peak) for measurement of the aversive thresholds. Alertness threshold was operationally defined as the lowest intensity producing episodes of movement arrest longer than $6 \mathrm{~s}$. Freezing threshold was defined as the lowest intensity producing immobility accompanied by at least two autonomic reactions such as urination, piloerection, defecation or exophthalmus. Escape threshold was defined as the lowest current intensity that produced running (gallop) or jumping in two successive ascending series of electrical stimulation. Animals with an escape threshold above $200 \mu \mathrm{A}$ (peak-to-peak) were excluded from the experiment.

The active avoidance cage consisted of a shuttle-box comprising two compartments of $30 \times 25 \times 25 \mathrm{~cm}$ with a 2.5 -cm high barrier between them and was equipped with 4 photoelectric cells equally spaced on the back wall. This arrangement allowed the detection of shuttle locomotion of the rat between the two compartments. The grid floor consisted of stainless steel rods spaced $1.2 \mathrm{~cm}$ apart. A 28-V light bulb and a loudspeaker were centered on the rear wall of each compartment of the chamber elevated $12 \mathrm{~cm}$ from the floor. The light was turned on and off noiselessly. The inferior colliculus stimulation was delivered by a protected wire lead that entered the conditioning chamber through a $2-\mathrm{cm}$ hole located in the top wall of the chamber. The rat was placed in the shuttlebox and had its brain electrode connected to a flexible wire cable, allowing ample movement inside the box. The cable, in turn, was connected to the stimulator by means of a mercury swivel mounted on the top of the experimental chamber. The brain stimulation was applied at a current intensity 5\% below the escape threshold previously determined in the open-field. The adequacy of this current intensity level for the escape response was chosen on the basis of previous studies from our laboratory $(5,10)$.

Fifteen minutes after the determination of the aversive threshold the animals were placed inside the shuttle-box (10 lux at the floor level) and left there for $30 \mathrm{~min}$ for habituation to the experimental context before the beginning of the session. During each conditioning trial, either a light (100 lux at the floor level) or a tone stimulus was applied to the cage $(70 \mathrm{~dB}, 3000 \mathrm{~Hz})$ for 20 $\mathrm{s}$ followed by electrical stimulation (AC, 60 $\mathrm{Hz}, 10 \mathrm{~s})$ delivered through the implanted electrode. Two successive trials were separated by a random interval of 10 to $50 \mathrm{~s}$. Each animal was submitted to only 1 session. An independent group of animals was submitted to sham surgery and to the same procedure as the tone group except that brain electrical stimulation was replaced with footshocks. Footshocks ( $0.6 \mathrm{~mA}$, alternating current) were delivered through the test cage floor by a constant current generator with a built-in scrambler (Albarsh Instruments Shocker, Porto Alegre, RS, Brazil). Whenever a rat passed from one compartment to the other during the warning stimulus (20 s) it avoided the brain stimulation (avoidance responses); if it changed compartments dur- 
ing electrical stimulation of the brain (10 s) then the stimulation was automatically terminated (escape responses). The latencies and the number of avoidance and escape responses were individually recorded. In order to estimate the occurrence of learning the session was divided into two parts of 25 trials each (first and second halves). Associative learning was considered to occur when the number of avoidance responses was higher than $60 \%$ of all possible responses in the second half of the session.

A PC computer connected through an interface to the experimental chamber controlled the presentation and termination of the warning and unconditioned stimuli, along with all data collection.

\section{Statistical analysis}

Data are reported as mean \pm SEM. Twoway ANOVA was applied to the three aversive thresholds - alertness, freezing and escape - recorded (factor 1) for the groups exposed to light or to a tone as the warning stimulus (factor 2). Latencies for avoidance and escape responses and frequency of avoidance responses in the present experiment were also subjected to two-way analysis of variance. Factor 1 refers to the first and second segments (25 trials each) of the sessions. Factor 2 corresponds to groups of pairing conditions (light or tone plus brain stimulation as well as tone plus footshock). Factors found to be significant were tested with Newman-Keuls comparisons.

\section{Histology}

Upon completion of the experiment, animals were deeply anesthetized with sodium pentobarbital and perfused intracardially with saline followed by formalin solution (10\%). The brains were then removed and left for three days in the formalin solution. Serial $50-\mu \mathrm{m}$ brain sections were cut with a microtome and stained with neutral red in order to localize the positions of the electrode tips according to the Paxinos and Watson atlas (12).

\section{Results}

The electrode placements for the subjects were mainly situated in the central nucleus of the inferior colliculus, as shown in Figure 1.

Two-way ANOVA applied to the data confirmed previous findings showing significant differences $(\mathrm{F} 2,42=32.27, \mathrm{P}<0.001)$ in the intensity of electric current applied to the inferior colliculus, in the production of alertness (47.81 \pm 2.54 (A, peak-to-peak)), freezing $(68.75 \pm 3.80(\mathrm{~A}))$, and escape responses $(98.12 \pm 6.15(\mathrm{~A}))(5,10)$. The groups (light or tone as a warning stimulus) did not differ in relation to the thresholds recorded $(\mathrm{F} 1,42=1.03, \mathrm{P}>0.05)$. Therefore, there was no statistically significant interaction between aversive thresholds and warning stimulus ( $\mathrm{P}$ $=0.80$ ).

Two-way ANOVA for repeated measures applied to latencies of avoidance responses revealed that the performances of the animals were significantly different depending on the segments of the sessions $(\mathrm{F} 2,21=42.01, \mathrm{P}<0.001)$. This analysis did not reveal significant differences among groups when performance in the whole session was considered $(\mathrm{F} 2,21=1.39, \mathrm{P}>0.05)$. However, the interaction between groups and halves barely reached significance (F2,21 $=3.43, \mathrm{P}=0.05)$. Post-hoc Newman-Keuls comparisons showed that the significance obtained was due to the differences in the latencies of avoidance responses between the two halves of the sessions for groups light + brain stimulation and tone + footshock $(\mathrm{P}<0.05$; Figure 2$)$. The same analysis performed on the number of avoidance responses to warning stimuli revealed significant differences among the groups tested $(\mathrm{F} 2,21=$ $6.70, \mathrm{P}<0.01)$ and between the two halves of the sessions $(\mathrm{F} 2,21=29.54, \mathrm{P}<0.001)$. There 


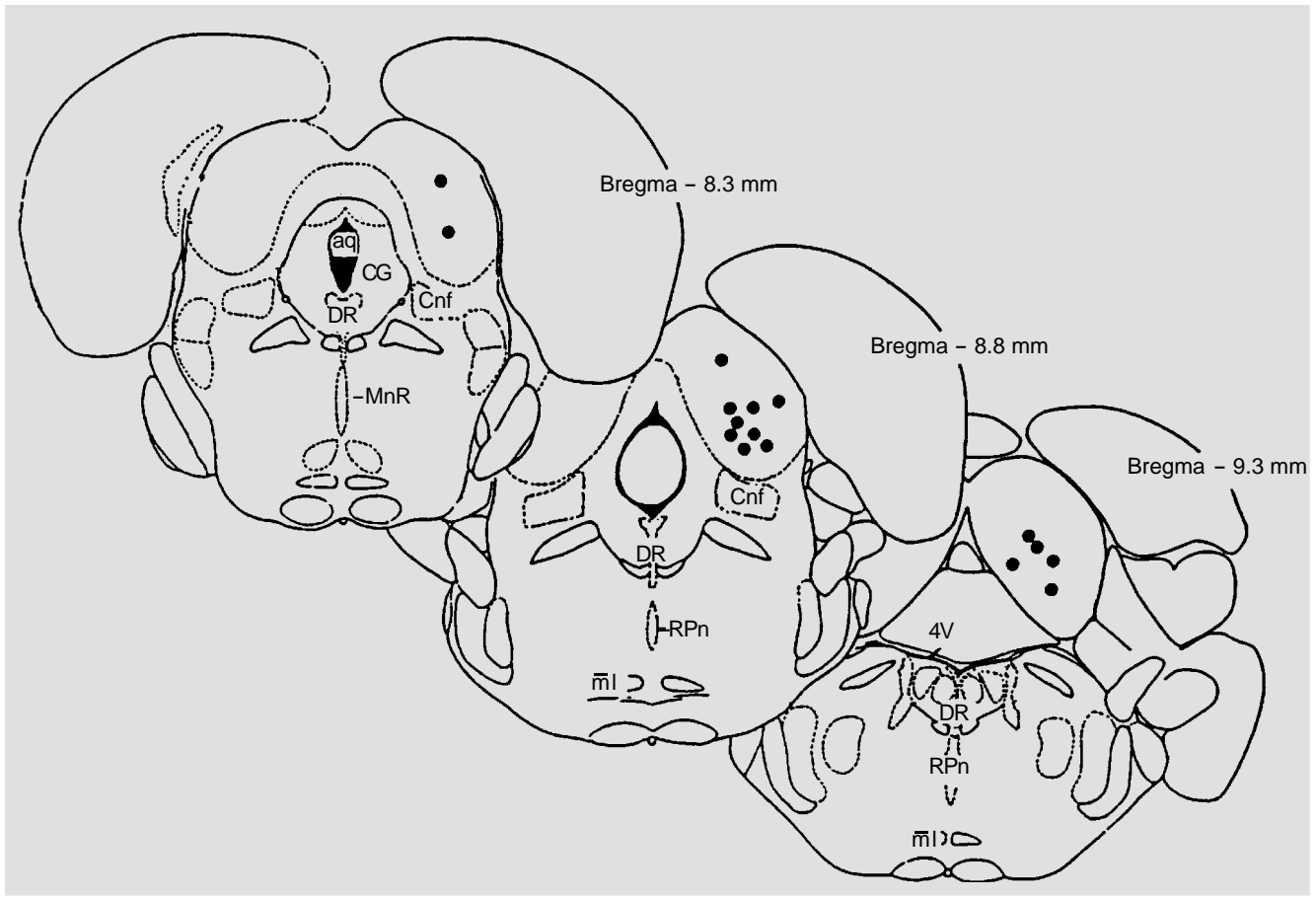

were no statistically significant interactions between groups or segments of the sessions $(\mathrm{F} 2,41=2.41, \mathrm{P}>0.05)$. Although all three groups increased their performances during the sessions the avoidance curves show a poorer performance of tone + brain stimulation in relation to the other groups (Figure 3). Actually, the mean values of the number of avoidance responses in the second halves of the sessions for this latter group $(11.2 \pm 1.1)$ did not reach the criterion of learning fixed at $60 \%$ of all possible responses as did the two other groups, light + brain stimulation $(19.6 \pm 1.6)$ and tone + footshock stimulation $(18.0 \pm 1.7)$. Furthermore, only one out of eight animals reached this criterion in the tone + brain stimulation group while this ratio was higher for the tone + footshock stimulation (6 of eight rats) and light + brain stimulation (7/8 animals) groups.

These data are consistent with the assertion that pairings of 20-s illumination with the houselight with inferior colliculus stimulation caused significant avoidance responses in the signaled avoidance learning paradigm used in this study. The same does not hold true for the pairing of tone to this stimulation.

Escape responses always occurred when the animals did not respond over the 20-s period of the warning stimulus. When we considered only these latencies of escape responses for the three groups tested, light + brain stimulation $(5.50 \pm 0.64 \mathrm{~s})$, tone + brain stimulation $(6.06 \pm 0.68 \mathrm{~s})$ and tone + footshock (4.25 $\pm 0.73 \mathrm{~s})$ ANOVA did not reveal any statistically significant differences among them $(\mathrm{F} 2,21=1.84, \mathrm{P}>0.05)$.

\section{Discussion}

The present study provides additional evidence for the involvement of the inferior colliculus in neural circuits subserving aversive reactions. Electrical stimulation of the inferior colliculus shares many of the aversive properties of the stimulation of structures classically considered as components of the brain aversion system, such as the dorsal periaqueductal gray matter, deep layers of the superior colliculus, medial hypothalamus and amygdala (4,14-16). This is consistent with the demonstration of ana-
Figure 1 - Location of sites on cross-sections from the rat brain atlas of Paxinos and Watson (12). Figures represent the atlas coordinates in millimeters posterior to the bregma. Black circles indicate the inferior colliculus sites where electrical stimulation was applied. aq = Aqueduct; $\mathrm{CG}=$ central gray; $\mathrm{DR}=$ dorsal raphe nucleus; $\mathrm{Cnf}=$ cuneiform nucleus; $\mathrm{ml}=$ medial lemniscus; $\mathrm{MnR}=$ median raphe nucleus; $4 \mathrm{~V}=$ fourth ventricle; $\mathrm{RPn}=$ raphe pontis nucleus. 
tomical connections between the inferior colliculus and these structures $(17,18)$.

The present results show that rats quickly learn to make a shuttling response in order to avoid or escape from electrical stimulation of the inferior colliculus. Thus, associative learning occurs using aversive states induced by inferior colliculus stimulation as the unconditioned stimulus, and light stimulation as the conditioned stimulus. So, the inferior colliculus stimulation functioned as negative reinforcement and light stimulation as a

Figure 2 - Latencies of avoidance responses during the first and second segments of two-way active avoidance sessions for rats submitted to conditioning by pairing light or a tone with brain stimulation and a tone with footshock. Each session consisted of 50 trials. The results were pooled and divided into two halves for analysis. Data are reported as mean \pm SEM for $\mathrm{N}=$ 8 rats in each group. ${ }^{*} \mathrm{P}<0.05$ compared to 1 st segment (Newman-Keuls comparisons).

Figure 3 - Number of avoidance responses during the first and second segments of two-way active avoidance sessions for rats submitted to conditioning by pairing light or a tone with brain stimulation and a tone with footshock. Each session consisted of 50 trials. The results were pooled and divided into two halves for analysis. Data are reported as mean \pm SEM for $\mathrm{N}=$ 8 rats in each group. ${ }^{*} \mathrm{P}<0.05$ compared to 1 st segment (Newman-Keuls comparisons).
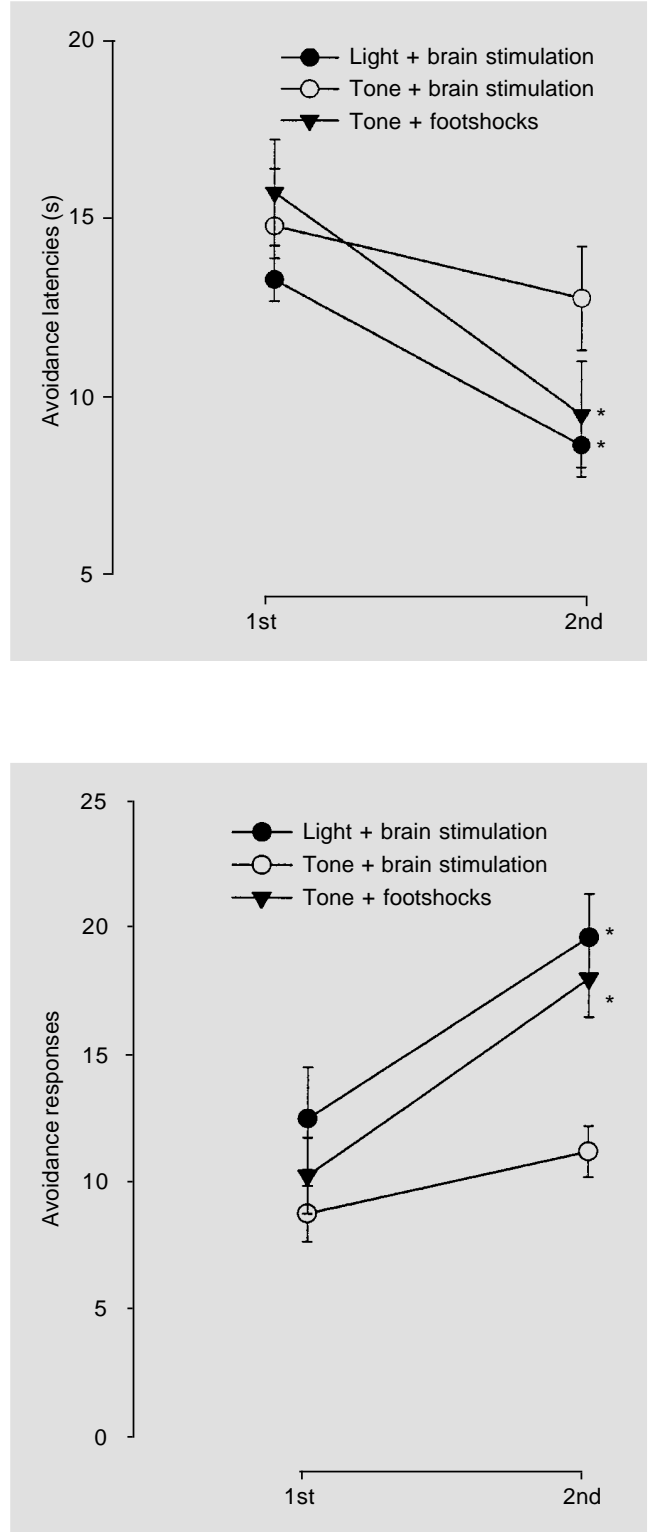

warning stimulus in the signaled two-way active avoidance paradigm used in this study. The rats increased their rate of responding in the presence of light over a number of trials. This effect may not be attributed to reflexive motor responses, since it was not observed when the neutral stimulus was presented alone, as demonstrated in a previous study from this laboratory (10). The pattern of results obtained in the present experiments with light as a warning stimulus parallels those which use a conditioned stimulus paired with stimulation of the dorsal periaqueductal gray $(19,20)$. It is likely that the inferior colliculus may be responsible for the elaboration of aversion and fear-like responses, together with other structures of the brain aversion system, such as dorsal periaqueductal gray matter and amygdala. As a matter of fact, there are important connections between the inferior colliculus and the dorsal periaqueductal gray matter (17). Furthermore, recent findings from this laboratory have shown that lesion of the central nucleus of the amygdala attenuates, while lesion of the basolateral complex enhances the aversive consequences of the electrical stimulation of the inferior colliculus (18).

In strong contrast to the association of light and inferior colliculus stimulation, acoustic stimulation paired with this stimulation does not function as a cue for rats to learn a shuttling response in order to avoid this aversive stimulation. These negative results may not be attributed to rupture of the tympanic membrane during stereotaxic surgery or the tone parameters used in this study. Supporting this statement, an independent group of animals submitted to the same procedure, except for the pairing of tone with footshock, showed reliable learning rates comparable to those from typical avoidance-escape procedures that utilize an auditory stimulus paired with electric shock as the unconditioned stimulus $(21,22)$. One possible explanation for these surprising results is that repeated electrical stimulation of 
the inferior colliculus leads to an increase of its reactivity to tone presentation. As a matter of fact, local microinjections of low doses of bicuculline into the inferior colliculus, which mimic the effects of its electrical stimulation (7), increase the responsiveness of this structure to sound (23). In support of this possibility it has been shown that repeated stimulation of the inferior colliculus resulted in modified sensitivity of its neural network and in a chronic susceptibility to spontaneous seizures (1). This shortcoming could lead to disturbances in the transmission of the to-be conditioned stimulus to a higher brain level, such as the amygdala, with which the inferior colliculus has an anatomical and functional relationship (18). Evidence showing that the steps of sensory processing are modulated during learning procedures (24, 25) supports this possibility. Interestingly, pairing tone with stimulation of the midbrain tectum, in which several sites of stimulation were located in the superior colliculus, a relay for visual information, produces a strong conditioning compared to a weak one when light was used as the conditioned stimulus (19).

Taken together, the present results suggest that rats learn to avoid the inferior colliculus stimulation when light is used as the warning stimulus. Since this does not occur when the neutral stimulus used is acoustic, it is likely that electrical stimulation of the inferior colliculus causes a disturbance in the transmission of auditory signals to higher brain structures, such as the amygdala, disturbing the conditioning process.

\section{References}

1. Bagri A, Sandner G \& Di Scala G (1991). Wild running and switch-off behavior elicited by electrical stimulation of the inferior colliculus: effects of anticonvulsant drugs. Pharmacology, Biochemistry and Behavior, 39: 683-688.

2. Brandão ML, Melo LL \& Cardoso SH (1993). Mechanisms of defense in the inferior colliculus. Behavioural Brain Research, 58: 49-55.

3. Silveira MCL, Sandner G \& Graeff FG (1993). Induction of Fos-immunoreactivity in the brain by exposure to the elevated plus-maze. Behavioural Brain Research, 56: 115-118.

4. Brandão ML, Cardoso SH, Melo LL, Motta V \& Coimbra NC (1994). Neural substrate of defensive behavior in the midbrain tectum. Neuroscience and Biobehavioral Reviews, 18: 339-346.

5. Melo LL, Cardoso SH \& Brandão ML (1992). Antiaversive action of benzodiazepines on escape behavior induced by electrical stimulation of the inferior colliculus. Physiology and Behavior, 51: 557562.

6. Schmitt $P$, Carrive $P$, Di Scala G, Jenck F, Brandão ML, Bagri A, Moreau JL \& Sandner G (1986). A neuropharmacological study of the periventricular neural substrate involved in flight. Behavioural Brain Research, 22: 181-190.

7. Brandão $M L$, Tomaz $C A B$, Leão Borges $P$
\& Bagri A (1988). Defence reaction induced by microinjection of bicuculline into the inferior colliculus. Physiology and Behavior, 44: 361-365.

8. Iwata J, Chida K \& LeDoux JE (1987). Cardiovascular responses elicited by stimulation of neurons in the central amygdaloid nucleus in awake but not anaesthetized rats resemble conditioned emotional responses. Brain Research, 418: 183-188.

9. LeDoux JE, Sakagushi A \& Reis DJ (1984). Subcortical efferent projections of the medial geniculate nucleus mediate emotional responses conditioned to acoustic stimuli. Journal of Neuroscience, 4: 683698.

10. Brandão $M L$, Troncoso $A C$, Melo LL \& Sandner G (1997). Active avoidance learning using brain stimulation applied to the inferior colliculus as negative reinforcement in rats: Evidence for latent inhibition. Neuropsychobiology, 35: 30-35.

11. MacIntosh NJ (1975). A theory of attention: variations in the associability of stimuli with reinforcement. Psychological Review, 82: 276-298.

12. Paxinos $G$ \& Watson $P$ (1986). The Rat Brain in Stereotaxic Coordinates. Academic Press, Sydney.

13. Marseillan RF (1977). A solid state sine wave stimulator. Physiology and Behavior, 19: 339-340.

14. Graeff FG (1990). Brain defense systems and anxiety. In: Roth M, Burrows GD \& Noyes R (Editors), Handbook of Anxiety. Vol. 3. Elsevier Science Publishers, Amsterdam, 307-354.

15. Hunsperger RW (1956). Affektreaktionen auf Elektrische Reizung im Hirnstamm der Katze. Helvetica Physiologica et Pharmacologica Acta, 14: 70-92.

16. Olds ME \& Olds J (1963). Approach-avoidance analysis of rat diencephalon. Journal of Comparative Neurology, 120: 259-295.

17. Kudo M \& Niimi K (1980). Ascending projections of the inferior colliculus in the cat: an autoradiographic study. Journal of Comparative Neurology, 191: 545-546.

18. Maisonnette SS, Kawasaki MC, Coimbra NC \& Brandão ML (1996). Effects of lesions of amygdaloid nuclei and substantia nigra on aversive responses induced by electrical stimulation of the inferior colliculus. Brain Research Bulletin, 40: 9398.

19. Di Scala G, Mana MJ, Jacobs WJ \& Phillips MJ (1987). Evidence of Pavlovian conditioned fear following electrical stimulation of the periaqueductal grey in the rat. Physiology and Behavior, 40: 55-63.

20. Di Scala G \& Sandner G (1989). Conditioned place aversion produced by microinjections of semicarbazide into the periaqueductal gray of the rat. Brain Research, 483: 91-97.

21. Freedman PE, Henessy JW \& Groner D 
(1974). Effects of varying active/passive shock levels in shuttle box avoidance in rats. Journal of Comparative and Physiological Psychology, 86: 79-84.

22. Gray JA (1977). Drug effects on fear and frustration: possible limbic site of action of minor tranquilizers. In: Iversen LL, Iversen SD \& Snyder SH (Editors), Handbook of Psychopharmacology. Vol. 8. Ple- num Press, New York, 433-529.

23. Bagri A, Sandner G \& Di Scala G (1989). Effects of unilateral microinjections of GABAergic drugs into the inferior colliculus on auditory evoked potentials and on audiogenic seizure susceptibility. Experimental Neurology, 104: 82-87.

24. Woody CD, Wang XF \& Gruen E (1994). Response to acoustic stimuli increases in the ventral cochlear nucleus after stimulus pairing. Neuroreport, 5: 513-514

25. Yamamoto $T$, Shimura $T$, Sako $N$ Yasoshima Y \& Sakai N (1994). Neural substrates for conditioned taste aversion in the rat. Behavioural Brain Research, 65: 123-137. 